\title{
Special issue on embedded systems in India
}

\author{
R. Varatharajan ${ }^{1}$
}

Published online: 28 November 2017

(C) Springer Science+Business Media, LLC, part of Springer Nature 2017

Asia-Pacific held the second place in the top revenue generating regions for embedded systems players in 2014 and is expected to retain its position till 2021. India is a major player in this region and is well on its way towards large scale implementation of modern embedded systems in automotive and consumer electronics industries.

The importance of India in the Asia-Pacific market for embedded systems prompted us to organize a special issue on embedded systems in India to capture significant research efforts ongoing in Indian institutions. For space reasons we are only able to include five papers, selected from multiple submissions.

The first paper, entitled Test Data Compression for Digital Circuits Using Tetrad State Skip Scheme, by Lokesh Sivanandam et al., proposes the Tetrad State Skip decomposition adequacy for various LFSR dimensions, showing excellent test data compression using less hardware.

The second paper by Joel Josephson et al., entitled A Novel Algorithm for Real-Time Framework in Multiprocessor Environment, presents a multiprocessor environment task scheduler for water tank control based on advanced particles swarm optimization algorithm, improving turnaround time, burst time and waiting time of multiprocessor environment when compared to other algorithms.

The third paper, TTHLS: An HLS tool for testable hardware generation, by Ravi et al., presents a novel methodology for the synthesis of testable hardware, by focusing on testability enhancements at the behavioural-level. The experimental results show that the proposed method reduces silicon area up to $9.38 \%$ and reduces power consumption up to $1.89 \%$.

The fourth paper, by Govindaraj Vellingiri, entitled An Improved Low Transition Test Pattern Generator for Low Power Applications, proposes a modified low transition linear feedback shift register (or MLT LFSR) circuit that reduces the total number of transitions without affecting the randomness. The experimental results show that there is a significant

R. Varatharajan

varathu21@yahoo.com

1 Department of Electronics and Communication Engineering, Sri Ramanujar Engineering College, Chennai, Tamil Nadu, India 
reduction in power consumption up to $36.2 \%$ for ISCAS' 85 combinational benchmark circuits and up to $10 \%$ for ISCAS' 89 benchmark sequential circuit.

The fifth paper, entitled A Novel Input Data Transition Aware Dynamic Voltage Scaling Based Low Power MAC Architecture for DSP Applications, by Hari Priya et al., proposes an input data transition-aware dynamic voltage scaling method applied to multiplieraccumulator architectures, which can achieve $28.19 \%$ power reduction when compared to conventional methods.

We would like to convey our sincere thanks to all the researchers for submitting their manuscripts and a special note of thanks to the reviewers, whose efforts have allowed the selection of good quality papers. We are also grateful to the Journal of Design Automation for Embedded Systems, for allowing us to divulge a selected sample of the ongoing research efforts on embedded systems in India. 